What are the required conditions for deltas at the shelf edge during rising sea level?

\author{
Jinyu Zhang, Ronald Steel and Cornel Olariu
}

Supplementary Materials

\title{
Summary of Contents
}

Figure DR1: Location of analysed shelf morphology used in this study

Table DR1: Sediment load and shelf morphology of 32 analysed shelves

Figure DR2: Relationship between shelf width and shelf gradient of 32 analysed shelves

References cited 


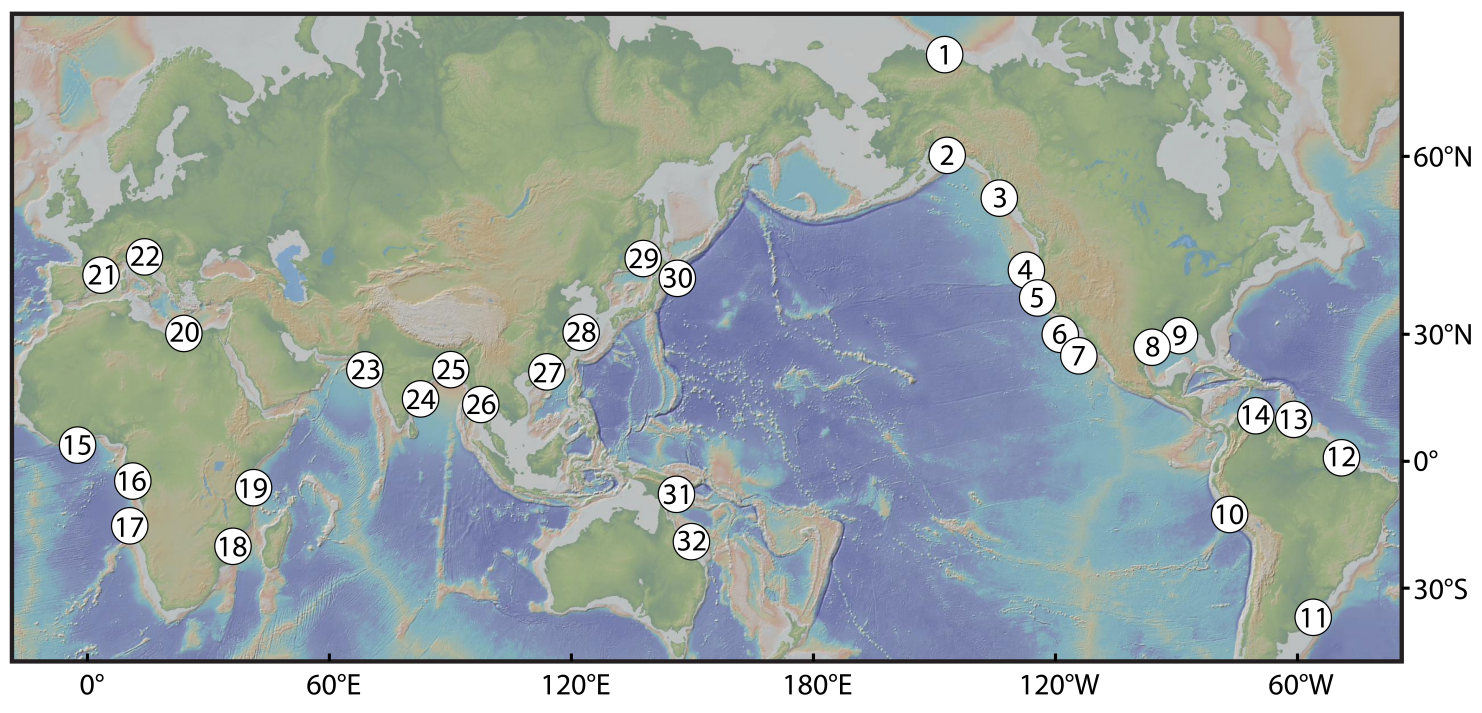

Figure DR1. Location of analysed shelf morphology used in this study. Refer to Table S1 for names and details. Base map is from GeoMapApp by Ryan et al. (2009). 


\begin{tabular}{|c|c|c|c|c|c|c|}
\hline $\begin{array}{l}\text { No. } \\
\text { (on } \\
\text { Fig }\end{array}$ & & $\begin{array}{c}\text { Sediment } \\
\text { load }\end{array}$ & $\begin{array}{c}\text { Average } \\
\text { shelf } \\
\text { width } \\
\end{array}$ & $\begin{array}{c}\text { Average } \\
\text { shelf } \\
\text { gradient }\end{array}$ & $\begin{array}{c}\text { Delta } \\
\text { system } \\
\text { width } \\
\end{array}$ & $\begin{array}{c}\text { Delta } \\
\text { foreset } \\
\text { gradient }\end{array}$ \\
\hline DR1) & River & $\mathrm{Mt} / \mathrm{yr}$ & $\mathrm{km}$ & $\circ$ & $\mathrm{km}$ & $\circ$ \\
\hline 1 & Mackenzie & 100 & 199 & 0.020 & 178 & 0.027 \\
\hline 2 & Copper & 70 & 123 & 0.070 & 25 & 0.391 \\
\hline 3 & Columbia & 15 & 43 & 0.237 & 51 & 0.320 \\
\hline 4 & Pajaro and Salinas & 2.6 & 17 & 0.448 & 38 & 0.792 \\
\hline 5 & $\begin{array}{l}\text { Ventura, Santa Clara } \\
\text { and Calleguas }\end{array}$ & 4.8 & 7.4 & 0.561 & 37 & 1.704 \\
\hline 6 & Santa Ana & 0.5 & 6.8 & 0.458 & 37 & 0.991 \\
\hline 7 & La Jolla* & 0.6 & 4.7 & 0.619 & 75 & 1.320 \\
\hline 8 & Brazos & 9.2 & 120 & 0.062 & 61 & 0.085 \\
\hline 9 & Mississippi & 400 & 133 & 0.060 & 250 & 0.485 \\
\hline 10 & Santa & 4.7 & 108 & 0.080 & 26 & 0.289 \\
\hline 11 & Parana & 90 & 111 & 0.050 & 265 & 0.054 \\
\hline 12 & Amazon & 1200 & 313 & 0.020 & 330 & 0.305 \\
\hline 13 & Orinoco & 210 & 122 & 0.040 & 235 & 0.143 \\
\hline 14 & Magdalena & 140 & 20 & 0.210 & 76 & 0.362 \\
\hline 15 & Niger & 190 & 82 & 0.070 & 305 & 0.407 \\
\hline 16 & Zaire & 43 & 87 & 0.080 & 62 & 0.195 \\
\hline 17 & Orange & 11 & 177 & 0.050 & 82 & 0.307 \\
\hline 18 & Limpopo & 26 & 32 & 0.180 & 24 & 0.261 \\
\hline 19 & Zambese & 48 & 76 & 0.060 & 96 & 0.174 \\
\hline 20 & Nile & 80 & 60 & 0.090 & 220 & 0.377 \\
\hline 21 & Rhone & 59 & 66 & 0.140 & 96 & 0.561 \\
\hline 22 & Danube & 67 & 97 & 0.080 & 99 & 0.154 \\
\hline 23 & $\begin{array}{l}\text { Indus } \\
\text { Krishna and }\end{array}$ & 250 & 168 & 0.060 & 240 & 0.101 \\
\hline 24 & Godavari & 128 & 18 & 0.300 & 220 & 0.194 \\
\hline 25 & Mahanadi and Gange & 1488 & 293 & 0.030 & 412 & 0.176 \\
\hline 26 & Irrawady & 360 & 175 & 0.034 & 151 & 0.061 \\
\hline 27 & Pearl & 80 & 347 & 0.030 & 76 & 0.044 \\
\hline 28 & Yangtze & 470 & 600 & 0.010 & 92 & 0.050 \\
\hline 29 & Ishikari & 1.8 & 43 & 0.135 & 27 & 0.524 \\
\hline 30 & Tokachi & 0.9 & 24 & 0.282 & 12 & 0.251 \\
\hline 31 & Fly & 80 & 166 & 0.041 & 104 & 0.110 \\
\hline 32 & Burdekin & 3.0 & 112 & 0.029 & 46 & 0.093 \\
\hline
\end{tabular}

*supplied by San Juan Capistrano, San Mateo, Santa Margarita, San Luis, and San Dieguito rivers

Table DR1. Sediment load from supplied river(s) and shelf morphology of 32 analysed shelves. Sediment load from supplied river(s) is collected by Milliman and Farnsworth (2013). Average shelf width, average shelf gradient, and delta system width are all measured following the approach of Olariu and Steel (2009) using the Global Multi-Resolution Topography dataset of Ryan et al. (2009). The delta foreset gradient is 
taken to be the steepest of either the subaerial delta (or shoreline) foreset slope or subaqueous delta slope (see definitions in Patruno et al., 2015).

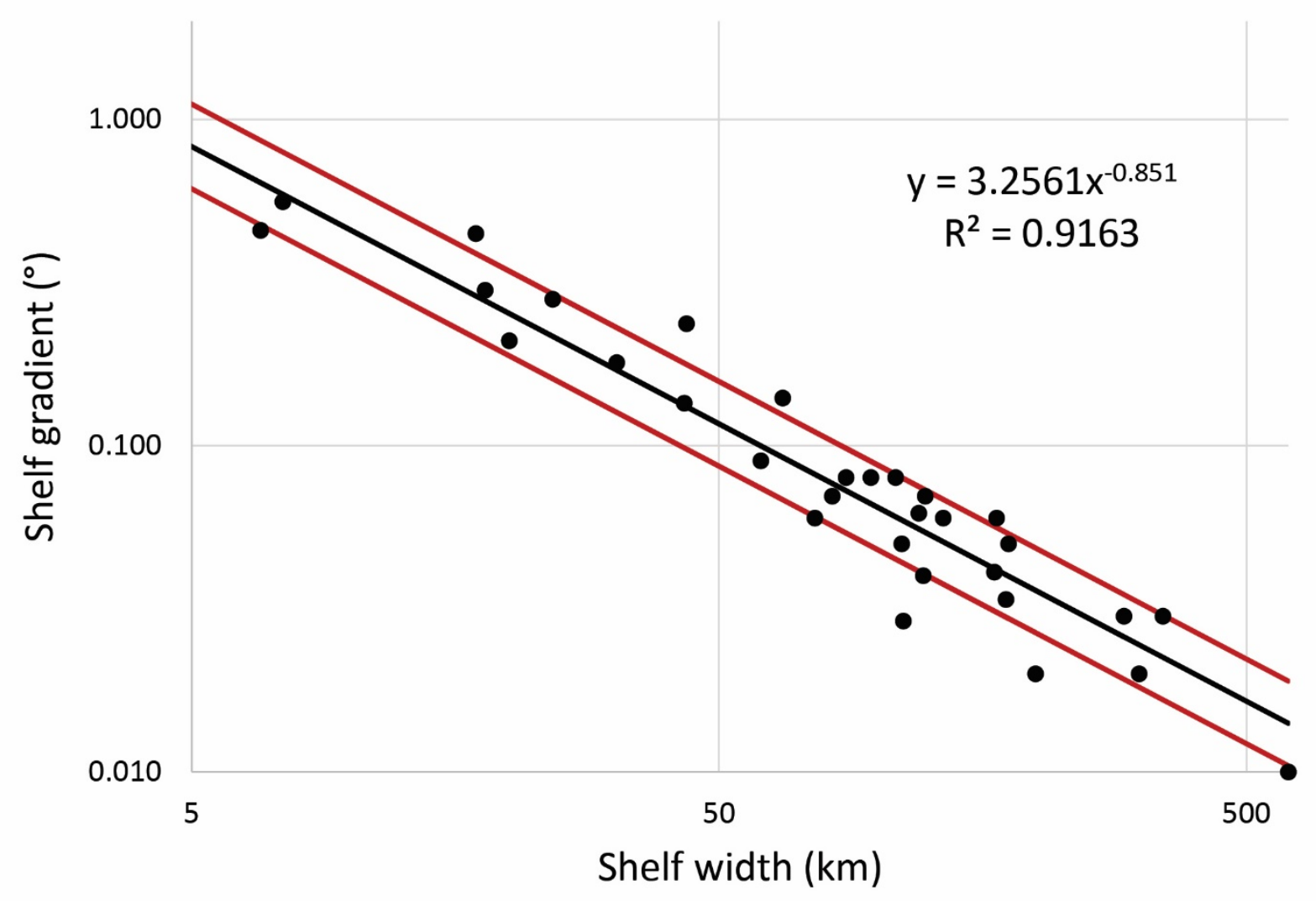

Figure DR2. The relationship between shelf width and shelf gradient of 32 analysed shelves. The best-fit relationship is in black and one standard deviation envelopes are in red.

\section{REFERENCES CITED}

Milliman, J. D., and Farnsworth, K. L., 2013, River Discharge to the Coastal Ocean: a Global Synthesis. Cambridge University Press.

Olariu, C., and Steel, R.J., 2009, Influence of point-source sediment-supply on modern shelf-slope morphology: Implications for interpretation of ancient shelf margins: Basin Research, v. 21, no. 5, p. 484-501, doi: 10.1111/j.1365-2117.2009.00420.x.

Patruno, S., Hampson, G.J., and Jackson, C.A.-L., 2015, Quantitative characterisation of deltaic and subaqueous clinoforms: Earth-Science Reviews, v. 142, p. 79-119, doi: 10.1016/j.earscirev.2015.01.004.

Ryan, W.B.F., and 11 others, 2009, Global multi-resolution topography synthesis: Geochemistry, Geophysics, Geosystems, v. 10, no. 3, doi: 10.1029/2008GC002332. 